\title{
Effect of Coriolis force on thermomagnetic convection in a ferrofluid saturating porous medium: A weakly nonlinear stability analysis
}

\author{
C.E. Nanjundappa ${ }^{\text {a }}$, I.S. Shivakumara ${ }^{\text {b }}$, H.N. Prakasha ${ }^{\text {c,* }}$ \\ a Department of Mathematics, Dr. Ambedkar Institute of Technology, Bangalore-560 056, India \\ ${ }^{\mathrm{b}}$ Department of Mathematics, Bangalore University, Bangalore-560 001, India

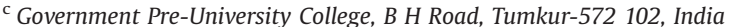

\section{A R T I C L E I N F O}

Article history:

Received 14 February 2014

Keywords:

Ferrofluid

Thermomagnetic convection

Coriolis force

Nonlinear stability

\begin{abstract}
A B S T R A C T
We investigate the influence of Coriolis force on the onset of thermomagnetic convection in ferrofluid saturating a porous layer in the presence of a uniform vertical magnetic field using both linear and weakly non-linear analyses. The modified Brinkman-Forchheimer-extended Darcy equation with Coriolis term has been used to describe the fluid flow. The linear theory based on normal mode method is considered to find the criteria for the onset of stationary thermomagnetic Convection and weakly non-linear analysis based on minimal representation of truncated Fourier series analysis containing only two terms has been used to find the Nusselt number $N u$ as functions of time. The range of thermal Rayleigh number $R$ beyond which the bifurcation becomes subcritical increases with increasing $\Lambda, D a^{-1}$ and $T a$. The global quantity of the heat transfer rate decreases by increasing the Taylor number $\mathrm{Ta}$. The results obtained, during the above analyses, have been presented graphically and the effects of various parameters on heat and mass transfer have been discussed. Finally, we have drawn the steady streamlines for various parameters.
\end{abstract}

(c) 2014 Published by Elsevier B.V.

\section{Introduction}

Thermomagnetic convection in magnetized ferrofluids saturating a porous layer is a topic of current technical importance since magnetic forces can be used to create circulation of coolant in small passages where natural convection is either absent or ineffective. The onset of thermomagnetic convection in a porous layer is investigated using Brinkman-Forchheimer-extended Darcy model. Most theoretical studies of ferroconvection in non-porous and porous domains have concentrated on the linearized equations and the studies undertaken on nonlinear convection are very sparse. Since the size of the ferromagnetic particles is small, ferrofluids can flow through porous media such as natural sediments or fractured rock due to gravitational, pressure gradient, capillary, and magnetic forces (Borglin et al. [1] and Oldenburg et al. [2]).

Schwab [3] has reported an analytical weakly nonlinear analysis of convective heat transfer in a ferrofluid of infinite magnetic susceptibility between free horizontal surfaces. Blennerhassett et al. [4] have analyzed weakly nonlinear thermo-convective stability of a ferrofluid, confined between rigid horizontal plates

\footnotetext{
* Corresponding author.

E-mail addresses: cenanju@hotmail.com (C.E. Nanjundappa), shivakumarais@gmail.com (I.S. Shivakumara), prakashahn83@gmail.com (H.N. Prakasha).
}

at different temperatures and subjected to a strong uniform external magnetostatic field in the vertical direction. Nonlinear convective roll cells that develop in thin layers of magnetized ferrofluids heated from above are examined in the limit as the wave number of the cells becomes large by Russell et al. [5]. Kaloni and Lou [6], Sunil and Mahajan [7] have performed nonlinear stability analysis of magnetized ferrofluid bounded by stress free boundaries and heated from below using the energy method. Recently, Laroze et al. [8] have reported theoretical and numerical results on thermally driven convection of a magnetic suspension by performing a truncated Galerkin expansion finding that the system can be described by a generalized Lorenz model.

The use of energy method to construct stability thresholds is crucial to assess whether the linear theory accurately encapsulates the physics of onset and the behavior of nonlinear ferroconvection in a porous medium. Straughan [9] has shown that the global nonlinear stability threshold for convection with a thermal nonequilibrium model is exactly the same as the linear instability boundary. Thermoconvective instability of magnetized ferrofluid saturating a porous medium has been studied using the global nonlinear stability analysis by Qin and Chadam [10] Sunil and Mahajan [11], while similar analysis has been carried out on the problem to include the local thermal nonequilibrium (LTNE) effects by Sunil et al. [12].

Blums et al. [13] have dealt with transport properties of ferrofluid nanoparticles in non-isothermal capillary-porous layer. 
Experimentally they have established that the temperature difference, which is applied across the layer, induces a thermo-osmotic pressure gradient directed toward increasing temperature. The measurement results are interpreted in a frame of phenomenology of linear irreversible thermodynamics.

Jyoti Prakash [14] has proved that the principle of exchange of stabilities is not, in general valid, for the case of free boundaries, in ferromagnetic convection, in a porous medium in the presence of a uniform vertical magnetic field and uniform rotation about the vertical axis and a sufficient condition is derived for the validity of this principle. Sekar et al. [15] have analyzed the Soret-driven ferro-thermo-convective instability of multicomponent fluid in an anisotropic porous medium heated from below and salted from above using the Brinkman model for various values of anisotropic parameter. Both stationary as well as oscillatory modes are considered in the study. The vertical anisotropy is shown to destabilize the system.

Jyoti Prakash and Sanjay Gupta [16] have investigated on arresting the complex growth rates in ferromagnetic convection with magnetic field dependent viscosity in a rotating ferrofluid layer. Straughan et al. [17] have analyzed Rotating porous convection with prescribed heat flux. Sekar et al. [18] have Studied the ferroconvection in fluids saturating a rotating densely packed porous medium. Nanjundappa et al. [19] have analysed penetrative ferroconvection via Internal Heating in a Saturated Porous Layer with Constant Heat Flux at the Lower Boundary. Nanjundappa et al. [20] was investigated the onset of coupled BénardMarangoni convection in a horizontal layer of ferrofluid with viscosity depending exponentially on temperature is investigated. The lower rigid and the upper free boundaries are assumed to be insulated to temperature perturbations and the free boundary at which the surface tension effects are accounted for is assumed to be non-deformable. The resulting eigenvalue problem is solved numerically using the Galerkin technique and also analytically by a regular perturbation technique with a wave number as a perturbation parameter. The analytical and numerically computed results are found to be in concurrence. The combined effect of magnetic number $\mathrm{M}_{1}$ and the viscosity parameter $\mathrm{B}$ is to reinforce together and to hasten the onset of Bénard-Marangoni ferroconvection compared to their presence in isolation. Nonetheless, the effect of increasing B also shows initially some stabilizing effect on the system depending on the strength of magnetic and buoyancy forces. In addition, the nonlinearity of fluid magnetization is found to have no influence on the criterion for the onset of BénardMarangoni ferroconvection.

Nonetheless, a different approach is followed in the present paper to study nonlinear thermomagnetic convection in a ferrofluid saturated porous medium. Instead of grappling with the full problem a simplified extended Lorenz model, put forward by Russell et al. [5], which reproduces qualitative features of the full system with remarkable fidelity is considered. This model problem, consisting of seven coupled nonlinear autonomous ordinary differential equations, are solved with sufficient accuracy by a combination of analytical and numerical techniques. The stability of bifurcating equilibrium solution is analyzed and heat transport is calculated in terms of Nusselt number which is missing in the previous studies.

\section{Formulation of the problem}

We consider an initially quiescent incompressible constant viscosity ferromagnetic fluid saturated horizontal porous layer of characteristic thickness $d$ in the presence of a uniform applied magnetic field $H_{0}$ in the vertical direction. The system is considered to be rotating about an axis with non-uniform rotation speed.
In particular, we assume that the rotation speed is varying sinusoidally with time. The lower surface is held at constant temperature $T_{l}$, while the upper surface is at $T_{u}\left(<T_{l}\right)$. A Cartesian co-ordinate system $(x, y, z)$ is used with the origin at the bottom of the porous layer and the z-axis directed vertically upward in the presence of gravitational field. The flow in the rotating ferrofluid saturating a porous medium is described by modified BrinkmanForchheimer-extended Darcy equation with fluid viscosity different from effective viscosity and the Boussinesq approximation on the density is made.

The basic equations governing the flow of an incompressible ferrofluid saturated porous medium under Oberbeck-Boussinesq approximation are:

$\nabla \times \vec{q}=0$

$$
\begin{aligned}
& \frac{\rho_{0}}{\varepsilon}\left[\frac{\partial \vec{q}}{\partial t}+\frac{1}{\varepsilon}(\vec{q} \times \nabla) \vec{q}\right] \\
& =-\nabla\left(p_{f}+\frac{\rho_{o}}{2}|\vec{\Omega} \times \vec{r}|^{2}\right)+\rho \vec{g}-\frac{\mu_{f}}{k} \vec{q}+\tilde{\mu}_{f} \nabla^{2} \vec{q} \\
& \quad+\mu_{0}(\vec{M} \times \nabla) \vec{H}+2 \rho_{o}(\vec{q} \times \vec{\Omega}) .
\end{aligned}
$$

$$
\begin{aligned}
& \varepsilon\left[\rho_{0} C_{V, H}-\mu_{0} \vec{H} \times\left(\frac{\partial \vec{M}}{\partial T}\right)_{V, H}\right] \frac{D T}{D t}+(1-\varepsilon)\left(\rho_{0} C\right) s \frac{\partial T}{\partial t}+\mu_{0} T\left(\frac{\partial \vec{M}}{\partial T}\right)_{V, H} \\
& \times \frac{D \vec{H}}{D t}=k_{t} \nabla^{2} T .
\end{aligned}
$$

$\rho=\rho_{0}\left[1-\alpha\left(T-T_{0}\right)\right]$

$$
\nabla \times \vec{B}=0
$$

$\nabla \times \vec{H}=0$ or $\vec{H}=\nabla \phi$

here, $P=p+\rho_{o}|\vec{\Omega} \times \vec{r}|^{2} / 2$ is the total pressure, $\vec{q}$ is the velocity of fluid particle, $\vec{\Omega}$ is the angular velocity, $\vec{r}$ is the position vector, $p$ is the fluid pressure, $\rho$ is the fluid density, $\rho_{0}$ is the reference density, $\vec{M}$ is the magnetization, $\vec{H}$ is the magnetic field intensity, $\vec{B}$ is the magnetic flux density, $\mu_{0}$ is the magnetic permeability of vacuum, $k_{t}$ is the thermal conductivity, $C_{V, H}$ is the specific heat at constant volume and magnetic field, $\alpha$ is the thermal expansion coefficient and $T_{a}=\left(T_{l}+T_{u}\right) / 2$ is the average temperature, $\mu_{f}$ and $\tilde{\mu}_{f}$ are the dynamic and effective viscosities, $k$ is the permeability of the porous medium and $\varepsilon$ is the porosity of the porous medium, $\nabla^{2}=\partial^{2} / \partial x^{2}+\partial^{2} / \partial y^{2}+\partial^{2} / \partial z^{2}$ is the Laplacian operator.

Further, $\vec{B}, \vec{M}$ and $\vec{H}$ are related by

$\vec{B}=\mu_{0}(\vec{M}+\vec{H})$

The local magnetization $\vec{M}$ is considered to be a function of $\vec{H}$ and $T$ in the form (Russell et al. [5])

$\vec{M}=\chi(T) \vec{H}$

where $\chi(T)$ is the magnetic susceptibility. This expression applies quite generally when the magnetic field is weak and we assume that $\chi(T)$ can be written in a form similar to the density as

$\chi(T)=\chi_{0}+\left(\frac{d \chi}{d T}\right)_{T_{a}}\left(T-T_{a}\right)$

The basic state is assumed to be quiescent and is given by

$\vec{q}_{b}=0$ 
$p_{b}(z)=p_{0}-\rho_{0} g z-\frac{1}{2} \rho_{0} \alpha g \beta z(z-d)-\frac{\mu_{0} M_{0} K \beta}{1+\chi_{0}} z-\frac{\mu_{0} K^{2} \beta^{2}}{2\left(1+\chi_{0}\right)^{2}} z(z-d)$

$T_{b}(z)=T_{0}-\beta(z-d / 2)$

$M_{b}(z)=M_{0}+\frac{K \beta}{\left(1+\chi_{0}\right)}(z-d / 2)$

$H_{b}(z)=H_{0}-\frac{K \beta}{\left(1+\chi_{0}\right)}(z-d / 2)$

$\rho_{b}=\rho_{0}[1+\alpha \beta(z-d / 2)]$

where, $\beta=\Delta T / d$ is the temperature gradient, $M_{0}=\chi_{0} H_{0}, K=$ $-H_{0}\left(d_{\chi} / d T\right)_{T_{a}}$ is the pyromagnetic coefficient, $\hat{k}$ is the unit vector in the $z$-direction and the subscript $b$ denotes the basic state. Here we have used the magnetic analogue of the Boussinesq approximation, that is, changes in $\chi(T)$ with temperature have been ignored except where multiplied by $H_{0}$.

To investigate the conditions under which the quiescent basic state is stable against small disturbances, we perturb the variables in the form

$\vec{q}=\vec{q}^{\prime}, p=p_{b}(z)+p^{\prime}, T=T_{b}(z)+\theta, \vec{M}=\vec{M}_{b}(z)+\vec{M}^{\prime}, \vec{H}=\vec{H}_{b}(z)+\vec{H}^{\prime}$

where $\overrightarrow{\mathrm{q}}^{\prime}=\left(\mathrm{u}^{\prime}, 0, \mathrm{w}^{\prime}\right), p^{\prime}, \theta, \overrightarrow{\mathrm{H}}^{\prime}=\left(\mathrm{H}_{\mathrm{x}}^{\prime}, 0, \mathrm{H}_{\mathrm{z}}^{\prime}\right)$ and $\overrightarrow{\mathrm{M}}^{\prime}=\left(\mathrm{M}_{\mathrm{x}}^{\prime}, 0, \mathrm{M}_{\mathrm{z}}^{\prime}\right)$ are perturbed quantities and are assumed to be small. Since we consider only two- dimensional motion, a stream function $\psi(x, z, t)$ is introduced such that $\vec{q}=u \hat{i}+w \hat{k}=\partial \psi / \partial z \hat{i}-\partial \psi / \partial x \hat{k}$. Also, the non-dimensional quantities are introduced in the form

$t^{\prime}=\left(d^{2} / \kappa\right) t *, \vec{q}^{\prime}=(\kappa / d) \vec{q} *, p^{\prime}=\left(\mu \kappa / d^{2}\right) p *, \theta^{\prime}=\beta d \theta *, \phi^{\prime}=\left(k \beta d^{2} / 1+\chi_{0}\right) \phi *$

where $\kappa=k /\left(\rho_{0} c\right)$ is the effective thermal diffusivity of the fluid, In view of the above, we note that $\overrightarrow{\mathrm{H}}$ can be written in the form

$\overrightarrow{\mathrm{H}}=H_{\mathrm{b}}(\mathrm{z}) \hat{\mathrm{k}}+\frac{\mathrm{K} \beta \mathrm{d}}{\left(1+\chi_{0}\right)} \nabla \phi *$

so that

$\overrightarrow{\mathrm{M}}=\left[\mathrm{M}_{\mathrm{b}}(\mathrm{z})-\mathrm{K} \beta \mathrm{d} \theta\right] \hat{\mathrm{k}}+\frac{\chi_{0} \mathrm{~K} \beta \mathrm{d}}{\left(1+\chi_{0}\right)} \nabla \phi *$

Substituting (15) in to the governing equations, eliminating the pressure by operating curl once and using (17) and (18) we obtain the following dimensionless equations after dropping the asterisks

$$
\begin{aligned}
& {\left[\frac{1}{\operatorname{Pr}} \frac{\partial}{\partial t}+D a^{-1}-\Lambda \nabla^{2}\right] \nabla^{2} \psi-\sqrt{T a} \frac{\partial v}{\partial z}+\left(R+R_{m}\right) \frac{\partial \theta}{\partial z}-R_{m} \frac{\partial^{2} \phi}{\partial z \partial x}} \\
& \quad=\frac{1}{\operatorname{Pr}} J\left(\psi, \nabla^{2} \psi\right)+R_{m} J\left(\theta, \frac{\partial \phi}{\partial z}\right)
\end{aligned}
$$

$\left[\frac{1}{\operatorname{Pr}} \frac{\partial}{\partial t}+D a^{-1}-\Lambda \nabla^{2}\right] v+\sqrt{T a} \frac{\partial \psi}{\partial z}=\frac{1}{\operatorname{Pr}} J(\psi, v)$

$\left[\frac{\partial}{\partial t}-\nabla^{2}\right] \theta+\frac{\partial \psi}{\partial x}=J(\psi, \theta)$

$\nabla^{2} \phi-\frac{\partial \theta}{\partial z}=0$

With boundary conditions

$\psi=\frac{\partial^{2} \psi}{\partial z^{2}}=\theta=\frac{\partial \phi}{\partial z}=\frac{\partial v}{\partial z}=0$ at $z=0,1$.

Here, $J(\ldots, \ldots)$ stands for the Jacobian, $R=\alpha \beta g d^{4} / A \nu \kappa$ is the thermal Rayleigh number, $D a=k / d^{2}$ is the Darcy number, $R m=\mu_{0} K^{2} \beta^{2} d^{4} / A \mu \kappa\left(1+\chi_{0}\right)$ is the magnetic Rayleigh number,
$T a=4 \Omega^{2} d^{4} / \nu^{2}$ is the Taylor number $\operatorname{Pr}=\nu / \varepsilon \kappa$ is the Prandtl number.

\section{The model}

To study the problem, a simplified model is constructed such that the linear results are identical with those for the full problem and the finite amplitude solution is exact to second order in the amplitude of the motion and appears to be qualitatively correct for larger amplitudes. For this purpose, following Russell et al. [5], the stream function, temperature fields and the magnetic potential are expanded as Fourier series in $x$ and $z$. From the physics of the problem it is clear that once convection sets in, the mean (or horizontally averaged) temperature and magnetic potential fields must be distorted by the convective motions. Hence, a minimal amplitude motion plus the distortion of temperature and magnetic potential fields is used as follows:

$\psi=A(t) \sin (\alpha x) \sin (\pi z)$,

$\theta=B(t) \cos (\alpha x) \sin (\pi z)+C(t) \sin (2 \pi z)$,

$v=D(t) \sin (\alpha x) \sin (\pi z)+E(t) \sin (2 \pi z)$,

$\phi=F(t) \cos (\alpha x) \cos (\pi z)+G(t) \cos (2 \pi z)$

where, the amplitudes $A(t)-G(t)$ are functions of time and must be determined. We note that (24a)-(24d) satisfy the respective boundary conditions. It is obvious that $\psi$ is minimally represented, since it is the simplest possible form for satisfying the boundary conditions; it is also the form of $\psi$ for the stability problem. The term $B(t) \cos (\alpha x) \sin (\pi z)$ is also minimal representation for $\theta$ and is included because it must balance the stream function term in the heat transport equation. The term $C(t) \sin (2 \pi z)$ represents the minimal representation for the distortion of the mean temperature field. The reason for the value 2 in the argument is that the mean temperature field is distorted by the convective term in the heat equation. Since both $\psi$ and $\theta$ have components proportional to $\sin (\pi z)$, this will force a $\sin (2 \pi z)$ dependence on the mean temperature. Similar remarks apply to vortices $v$ as well as to magnetic potential field $\phi$. Substituting (24) into (19)-(22) and equating the coefficients of like terms, we obtain the following nonlinear autonomous system of ordinary differential equations for the amplitudes:

$$
\begin{aligned}
\frac{d A}{d t} & =-\operatorname{Pr}\left(\left(D a^{-1}+\Lambda \delta^{2}\right) A+\frac{\alpha}{\delta^{2}}\left(R+R_{m}\right) B-\frac{\pi}{\delta^{2}} \sqrt{T a} D+\frac{\pi}{\delta^{2}} \alpha R_{m} F\right. \\
& \left.+\frac{\pi^{2}}{\delta^{2}} \alpha R_{m} C F-2 \frac{\pi^{2}}{\delta^{2}} \alpha R_{m} B G\right)
\end{aligned}
$$

$\frac{d B}{d t}=-\delta^{2} B-\alpha A-\pi \alpha A C$

$\frac{d C}{d t}=-4 \pi^{2} C+\frac{\pi}{2} \alpha A B$

$\frac{d D}{d t}=-\left(D a^{-1}+\Lambda \delta^{2}\right) \operatorname{Pr} D-\pi \operatorname{Pr} \sqrt{T a} A+\pi \alpha A E$

$\frac{d E}{d t}=-\operatorname{Pr} D a^{-1} E-4 \alpha^{2} \Lambda \operatorname{Pr} E-\frac{\pi}{2} \alpha A D$

$F=\frac{-\pi}{\delta^{2}} B$

$G=\frac{-C}{2 \pi}$

where $\delta^{2}=\pi^{2}+\alpha^{2}$. Eqs. (25a)-(25e) are the basic set that will be studied in this Chapter. These equations possess two significant 
properties. First, the divergence of the flow in phase space,

$\frac{\partial \dot{A}}{\partial A}+\frac{\partial \dot{B}}{\partial B}+\frac{\partial \dot{C}}{\partial C}+\frac{\partial \dot{D}}{\partial D}+\frac{\partial \dot{E}}{\partial E}=-\operatorname{Pr}\left(3 D a^{-1}+2 \Lambda\left(\delta^{2}+2 \alpha^{2}\right)\right)-\left(\delta^{2}+4 \pi^{2}\right)$

is always negative and so the solutions are attracted to a set of measure zero in the phase space: this may be a fixed point, a limit cycle or a strange attractor. The dot above a quantity denotes the derivative with respect to time $t$. Second, the equations have an important symmetry as they are unchanged under the transformation

$(A, B, C, D, E) \rightarrow(-A,-B, C,-D, E)$.

The set of nonlinear ordinary differential equations given by (25a)-(25e) is complicated to solve for the general time dependent fields. However, we can look at the steady-state solution to the system. It turns out that this information is very useful to have because it predicts that a finite-amplitude steady solution bifurcates subcritically or supercritically and also helps in finding the amount of heat transfer. Setting the left hand sides of (25a)-(25e) equal to zero and using (25f)-(25g), we obtain

$\left(D a^{-1}+\Lambda \delta^{2}\right) \delta^{4} A+\alpha \delta^{2} B R-\pi \delta^{2} \sqrt{T a} D+\alpha^{3}(1+\pi C) R_{m} B=0$

$\delta^{2} B+\alpha A+\pi \alpha A C=0$

$8 \pi^{2} C-\pi \alpha A B=0$

$\left(D a^{-1}+\Lambda \delta^{2}\right) \operatorname{Pr} D+\pi \operatorname{Pr} \sqrt{T a} A-\pi \alpha A E=0$

$\left(2 D a^{-1}+8 \Lambda \alpha^{2}\right) \operatorname{Pr} E+\pi \alpha A D=0$

\subsection{Stationary convection}

Neglecting the nonlinear terms from the system (28a-e) and eliminating the amplitude $B$ from (28a) leads to an expression for the magnetic Rayleigh number at which the stationary convection sets in. That is,

$R_{m}=\frac{\left(D a^{-1}+\Lambda \delta^{2}\right) \delta^{6}}{\alpha^{4}}+\frac{\pi^{2} \delta^{4}}{\alpha^{4}\left(D a^{-1}+\Lambda \delta^{2}\right)} T a-\frac{\delta^{2}}{\alpha^{2}} R=R_{m}^{s}$

It is noted that $R_{m}^{s}$ attains its critical value $R_{m c}^{s}$ at $\alpha^{2}=\alpha_{c}^{2}$ where $\alpha_{c}^{2}$ satisfies the equation

$b_{1}\left(\alpha_{c}^{2}\right)^{6}+b_{2}\left(\alpha_{c}^{2}\right)^{5}+b_{3}\left(\alpha_{c}^{2}\right)^{4}+b_{4}\left(\alpha_{c}^{2}\right)^{3}+b_{5}\left(\alpha_{c}^{2}\right)^{2}+b_{6}\left(\alpha_{c}^{2}\right)+b_{7}=0$

where,

$b_{1}=2 \pi^{3} \Lambda^{2}$

$b_{2}=\Lambda^{4} D a^{-1}+4 \pi^{2} \Lambda^{2}\left(\Lambda^{3}+\pi^{3}\right)$

$b_{3}=2 \pi^{2} D a^{-1}\left(\pi D a^{-1}+\Lambda^{4}\right)+2 \pi^{4} \Lambda^{2}\left(4 \Lambda^{3}+\pi^{3}\right)$

$b_{4}=\Lambda^{2} D a^{-1}\left(D a^{-1}\left(D a^{-1}+4 \Lambda \pi^{2}\right)-2 \pi^{4} \Lambda^{2}\right)+\pi^{2} \Lambda^{3}(\Lambda R-T a)$

$b_{5}=2 \pi^{2} \Lambda^{2}\left[D a^{-1}\left(T a-4 \pi^{4} \Lambda^{2}\right)-2 \pi^{2} \Lambda T a-5 \pi^{6} \Lambda^{3}+\pi^{2} \Lambda^{2} R\right]$

$b_{6}=\pi^{2} \Lambda\left[\begin{array}{l}D a^{-1}\left(4 \pi^{2} \Lambda T a-7 \pi^{6} \Lambda^{3}+D a^{-1}\left(\Lambda R-4 T a-4 \pi \Lambda^{5}-3 \pi^{2} \Lambda D a^{-1}\right)\right) \\ -\pi^{2} \Lambda^{2}\left(8 \pi^{6} \Lambda^{2}+5 \pi^{2} T a-\Lambda^{3} R\right)\end{array}\right]$

$b_{7}=\pi^{2} \Lambda\left[D a^{-1}\left(4 \pi^{2} \Lambda T a-7 \pi^{6} \Lambda^{3}+D a^{-1}\left(\Lambda R-4 T a-4 \pi \Lambda^{5}\right.\right.\right.$ $\left.\left.\left.-3 \pi^{2} \Lambda D a^{-1}\right)\right)-\pi^{2} \Lambda^{2}\left(8 \pi^{6} \Lambda^{2}+5 \pi^{2} T a-\Lambda^{3} R\right)\right]$

It is interesting to look for some known results from (29) under limiting cases. For the non-porous domain case $\left(\mathrm{Da}^{-1}=0, \Lambda=1\right)$, (34) reduces to

$R_{m}=\frac{\delta^{8}}{\alpha^{4}}+\frac{\pi^{2} \delta^{2}}{\alpha^{4}} T a-\frac{\delta^{2}}{\alpha^{2}} R$
For the non-rotating system in a non-porous ferrofluid layer ( $\left.T a=0=D a^{-1}, \quad \Lambda=1\right)$, Eq. (31) reduces to

$R_{m}=\frac{\delta^{8}}{\alpha^{4}}-\frac{\delta^{2}}{\alpha^{2}} R$

The above expression coincides with Russell et al. [5] and it attains the critical value when the critical wave number satisfies the equation.

$2\left(\alpha_{c}^{2}\right)^{4}+4 \pi^{2}\left(\alpha_{c}^{2}\right)^{3}+\left(\pi^{2} R-4 \pi^{6}\right)\left(\alpha_{c}^{2}\right)-2 \pi^{8}=0$.

Further, for an ordinary viscous fluid medium case $\left(R_{m}=0\right)$, Eq. (29) gives an expression for $R$ in the form

$R=\frac{\delta^{6}}{\alpha^{2}}+\frac{\pi^{2}}{\alpha^{2}} T a$.

The above result coincides with the one obtained by G Veronis [21]. It is thus observed that the model equations considered gives the results of linear stability theory results which are identical with those for the full problem.

\subsection{Steady finite amplitude convection}

The linear stability theory does not give any information about the stability of bifurcating finite amplitude solution and also the heat transfer. This can be achieved by considering the nonlinear terms in the equations. Expressing the amplitudes $B, C, D$ and $E$ in terms $A$ using (28b)-(28e), we obtain

$B=-\frac{\alpha A}{\delta^{2}+\alpha^{2} A^{2} / 8}$

$C=\frac{\alpha}{8 \pi} A B$

$D=-\frac{2 \pi \sqrt{T a} \operatorname{Pr}^{2}\left(D a^{-1}+4 \Lambda \alpha^{2}\right) A}{\pi^{2} \alpha^{2} A^{2}+2 \operatorname{Pr}^{2}\left(D a^{-1}+4 \Lambda \alpha^{2}\right)\left(D a^{-1}+\Lambda \delta^{2}\right)}$

$E=-\frac{\pi \alpha}{\operatorname{Pr}\left(2 D a^{-1}+8 \Lambda \alpha^{2}\right)} A D$

From (25a), we now obtain an equation for the amplitude $A$ in the form

$A\left[b_{0}\left(A^{2}\right)^{3}+b_{1}\left(A^{2}\right)^{2}+b_{2}\left(A^{2}\right)+b_{3}\right]=0$

where

$\Delta_{1}=D a^{-1}+\Lambda \delta^{2} \Delta_{2}=D a^{-1}+4 \Lambda \alpha^{2} b_{0}=\pi^{2} \alpha^{6} \delta^{2} \Delta_{1}$

$b_{1}=16 \pi^{2} \alpha^{4} \delta^{4} \Delta_{1}+2 \alpha^{4} \operatorname{Pr}^{2} \Delta_{1}^{2} \Delta_{2} \delta^{2}+2 \pi^{2} \alpha^{4} \operatorname{Pr}^{2} \Delta_{2} T a-8 \pi^{2} \alpha^{6} R$

$b_{2}=64 \pi^{2} \alpha^{2} \delta^{6} \Delta_{1}+32 \alpha^{2} \Delta_{1}{ }^{2} \Delta_{2} \delta^{4} \operatorname{Pr}^{2}-64 \pi^{2} \alpha^{4} \delta^{2} R-16 \alpha^{4} \Delta_{1} \Delta_{2} \operatorname{Pr}^{2} R$

$+32 \pi^{2} \alpha^{2} \delta^{2} \operatorname{Pr}^{2} \Delta_{2} T a-64 \pi^{2} \alpha^{6} R_{m}$

$b_{3}=128 \Delta_{1}{ }^{2} \Delta_{2} \delta^{6} \operatorname{Pr}^{2}-128 \Delta_{1} \Delta_{2} \alpha^{2} \delta^{2} \operatorname{Pr}^{2} R-128 \Delta_{1} \Delta_{2} \operatorname{Pr}^{2} \alpha^{4} R_{m}$

$$
+128 \pi^{2} \Delta_{2} \delta^{4} \operatorname{Pr}^{2} T a
$$

The solution $A=0$ corresponds to pure conduction, which we know to be a possible solution though it is unstable when the Rayleigh number $R$ is sufficiently large. The remaining solutions are given by

$$
\begin{aligned}
\pi^{2} \alpha^{6} \delta^{2} \Delta_{1}\left(A^{2}\right)^{3}+\left\{16 \pi^{2} \alpha^{4} \delta^{4} \Delta_{1}+2 \alpha^{4} \operatorname{Pr}^{2} \Delta_{1}{ }^{2} \Delta_{2} \delta^{2}\right. \\
\left.+2 \pi^{2} \alpha^{4} \operatorname{Pr}^{2} \Delta_{2} T a-8 \pi^{2} \alpha^{6} R\right\}\left(A^{2}\right)^{2} \\
+\left\{\begin{array}{l}
64 \pi^{2} \alpha^{2} \delta^{6} \Delta_{1}+32 \alpha^{2} \Delta_{1}{ }^{2} \Delta_{2} \delta^{4} \operatorname{Pr}^{2}-64 \pi^{2} \alpha^{4} \delta^{2} R-16 \alpha^{4} \Delta_{1} \Delta_{2} \operatorname{Pr}^{2} R \\
+32 \pi^{2} \alpha^{2} \delta^{2} \operatorname{Pr}^{2} \Delta_{2} T a-64 \pi^{2} \alpha^{6} R_{m}
\end{array}\right\}\left(A^{2}\right) \\
-128 \Delta_{1} \Delta_{2} \alpha^{4} \operatorname{Pr}^{2}\left(R_{m}-R_{m}^{s}\right)=0
\end{aligned}
$$


In the neighborhood of $R_{m}^{s}$ there is a triplet of steady solutions, one of which is the static solution (which is always unstable for $R_{m}>R_{m}^{s}$ ). The other two are finite-amplitude solutions which, owing to the symmetry of (28a-e), differ only in the sign of $A, B$ and $D$. To investigate the behavior of steady finite amplitude solution in the neighborhood of $R_{m}$, we use modified perturbation theory. Accordingly, $R_{m}$ is expanded in terms of the amplitude Accordingly, $R_{m}$ is expanded in terms of the amplitude $A^{2}<<1$ in the form

$R_{m}=R_{m 0}+\left(\frac{A^{2}}{8}\right) R_{m 2}+\cdots$

Eq. (38) is substituted back into (37) and the coefficients of like powers of $A^{2}$ are collected.

At zeroth order in $A^{2}$, the results of linear stability are retrieved and is found that

$R_{m 0}=R_{m}^{S}=\frac{\Delta_{1} \delta^{6}}{\alpha^{4}}+\frac{\pi^{2} \delta^{4}}{\alpha^{4} \Delta_{1}} T a-\frac{\delta^{2}}{\alpha^{2}} R$

\section{a}

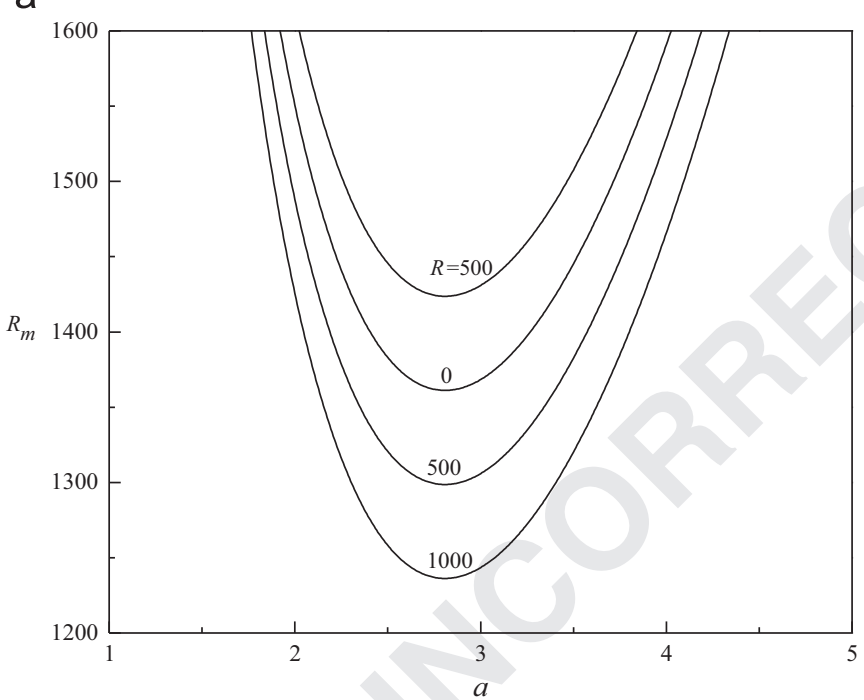

b

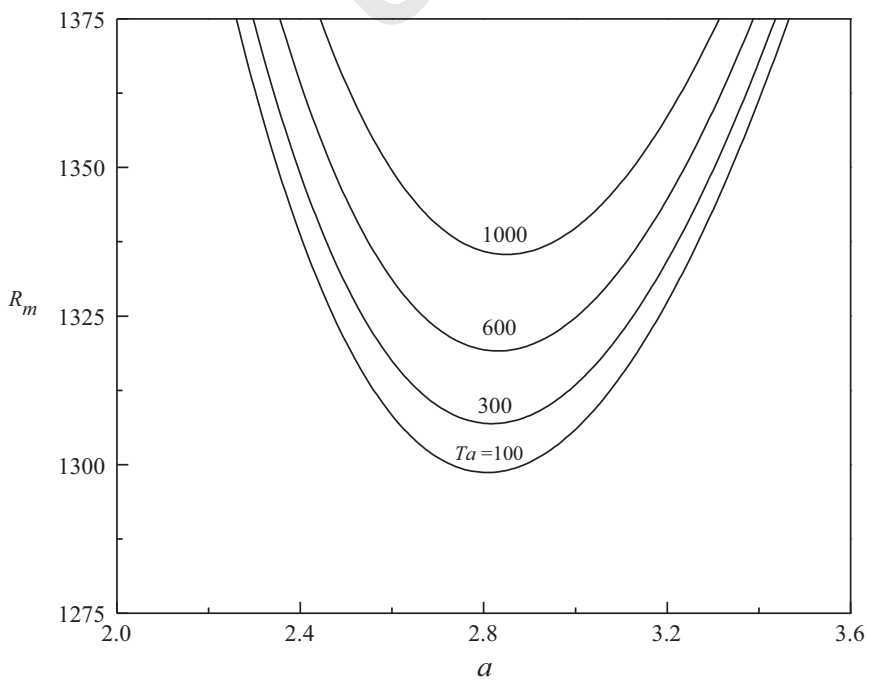

Fig. 2. Neutral curves for different values of (a) $R$ with $T a=100$ and (b) $T a$ with $R=500$ for $\operatorname{Pr}=10, D a^{-1}=100$ and $\Lambda=2$.
At the first order in $A^{2}$, we find that

$$
R_{m 2}=\frac{\Delta_{1} \delta^{4}}{4 \alpha^{2}}+\frac{\pi^{2} \delta^{2}}{4 \Delta_{1} \alpha^{2}} T a-\frac{\pi^{4} \delta^{4}}{2 \alpha^{4} \Delta_{1}^{2} \Delta_{2} \operatorname{Pr}^{2}} T a-\frac{R}{8}
$$

This is the first non-trivial steady finite-amplitude magnetic Rayleigh number. It is observed that $R_{m 2}$ may be either positive or negative depending on the value of thermal Rayleigh number. The finite-amplitude solution is said to be stable (bifurcation is supercritical) if $R_{m 2}>0$ and said to be unstable (bifurcation is subcritical) if $R_{m 2}<0$. From (40) it is seen that for negative values of $R$,

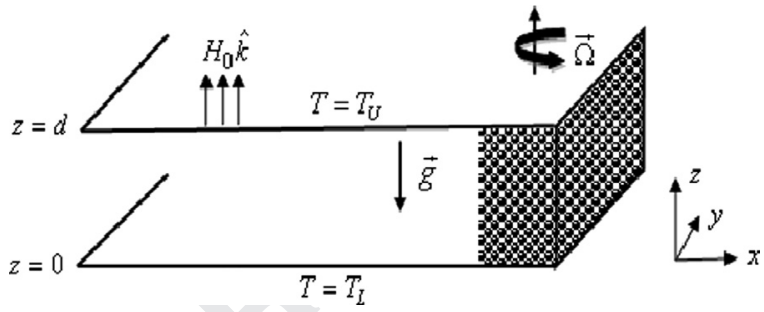

Fig. 1. Physical configuration.

a

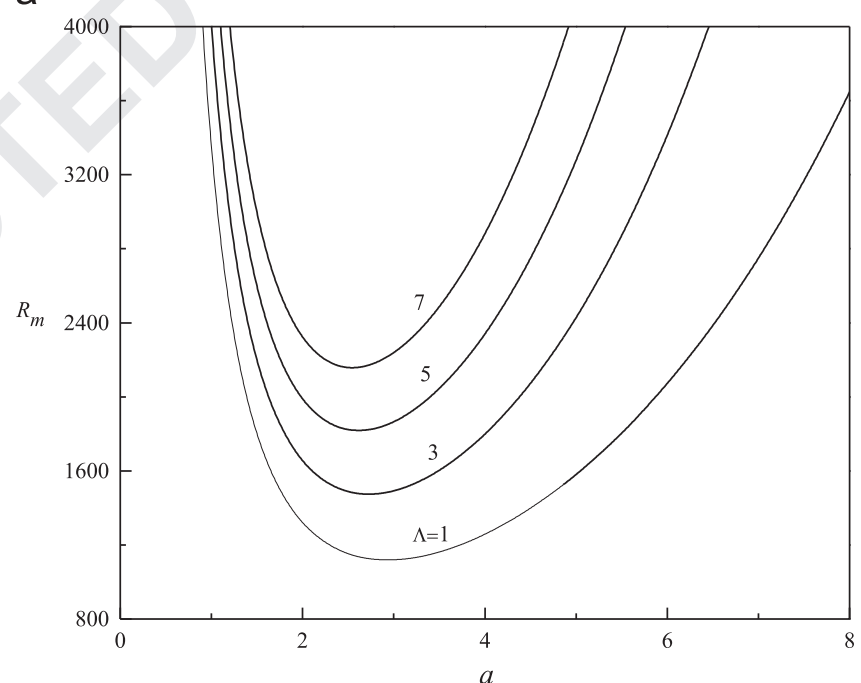

b

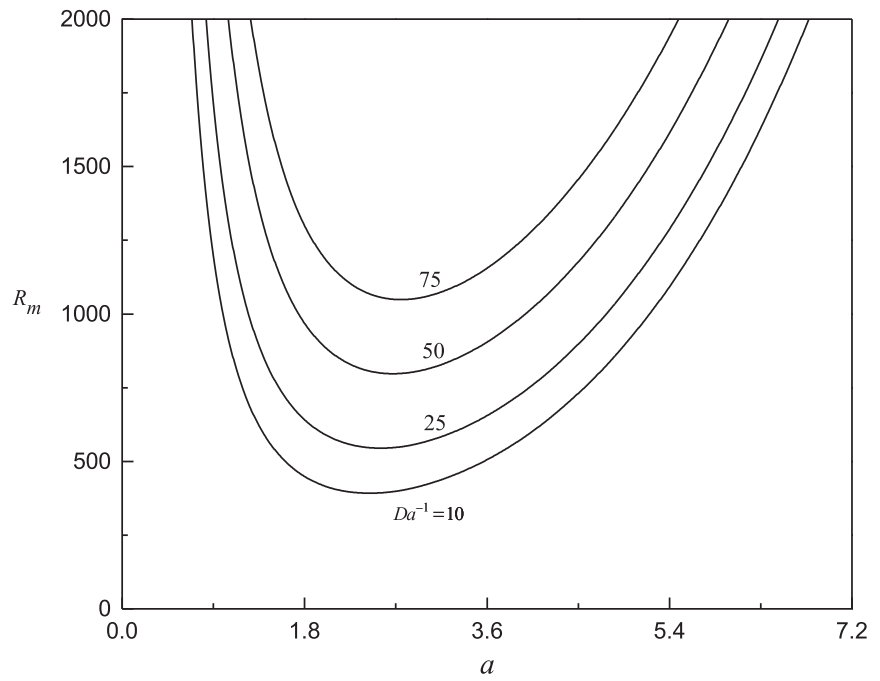

Fig. 3. Neutral curves for different values of (a) $\Lambda$ and (b) $D a^{-1}$ for $T a=100$, $R=500$ and $\operatorname{Pr}=10$ 
the finite-amplitude solution is always stable and hence subcritical instability is not possible.

\section{Heat transport}

The vigor of convection is measured in terms of heat and mass transfer. If $\bar{H}$ is the rate of heat transport per unit area then

$\bar{H}_{t}=-\kappa\left\langle\frac{\partial T}{\partial z}\right\rangle_{z=0}$

where the angular brackets correspond to a horizontal average. Substituting

$T=T_{0}-\beta d\left(z-\frac{1}{2}\right)+\theta(x, z, t)$.

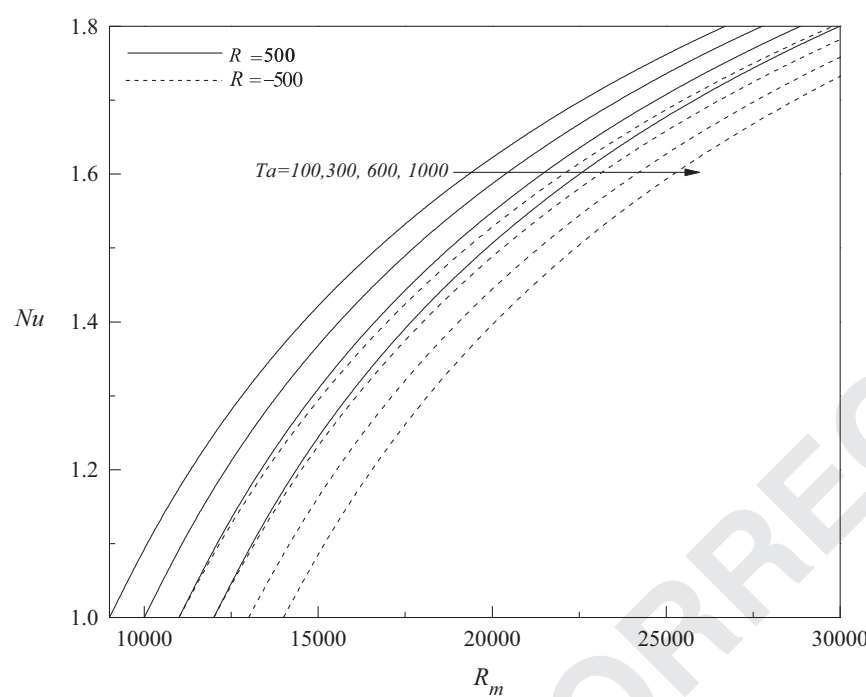

Fig. 4. Variation of $N u$ with $R_{m}$ for different values of $T a$ when $\operatorname{Pr}=10, \Lambda=2$ and $D a^{-1}=100$.

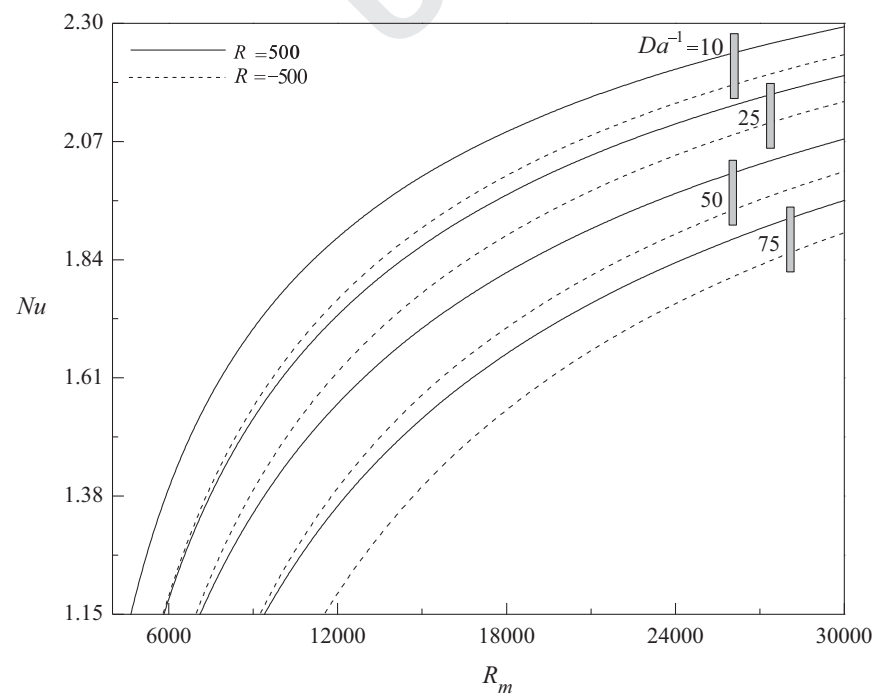

Fig. 5. Variation of $N u$ with $R_{m}$ for different values of $D a^{-1}$ when $\operatorname{Pr}=10, T a=100$ and $\Lambda=2$. in the above equation, performing the integration and using (16), we obtain an expression for the Nusselt number $\mathrm{Nu}$ in the form

$N u=\frac{\bar{H} d}{\kappa \Delta T}=1-2 \pi C=1+2 \frac{\alpha^{2} A^{2}}{\left(8 \delta^{2}+\alpha^{2} A^{2}\right)}$

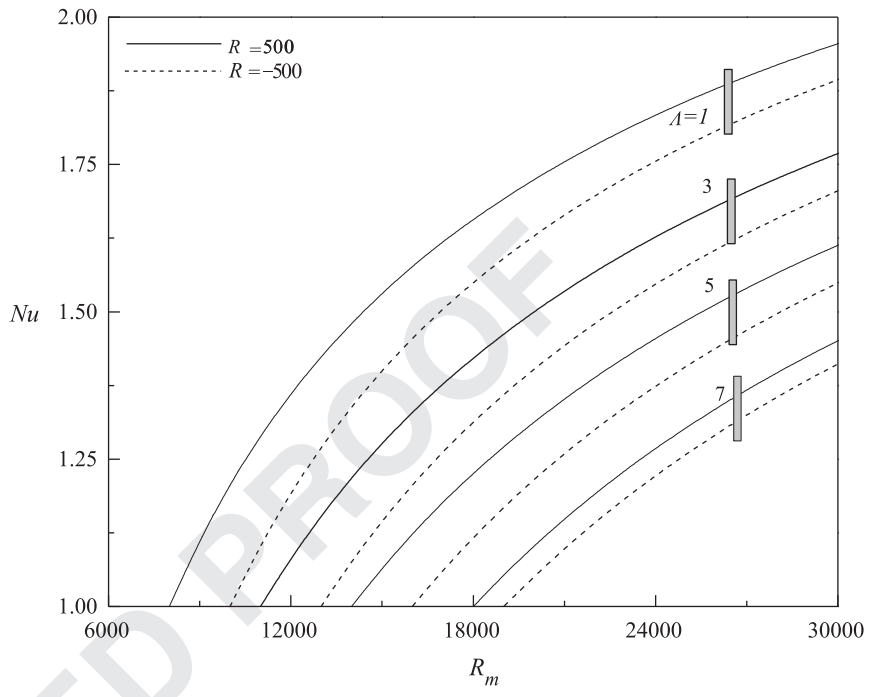

Fig. 6. Variation of $N u$ with $R_{m}$ for different values of $\Lambda$ when $\operatorname{Pr}=10, T a=100$ and $D a^{-1}=100$.

a

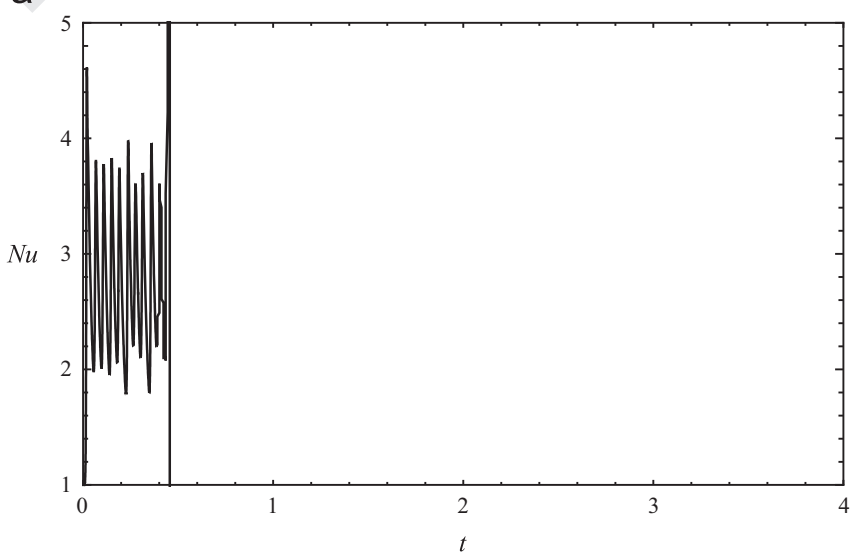

b

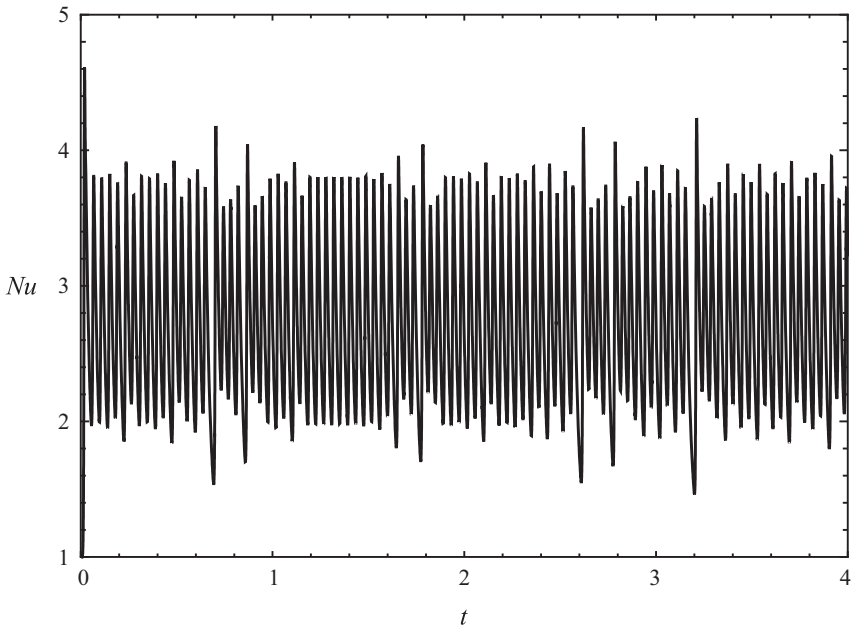

Fig. 7. Variation of Nusselt number with time $t$ for two of values of (a) $T a=0$ and (b) $T a=100$, when $\operatorname{Pr}=7, D a^{-1}=25, \Lambda=2, R_{m}=40$ and $R=80000$. 
It is observed that in the absence of convection the heat transfer is only by conduction and in that case $N u=1$.

\section{Results and discussion}

Two-dimensional ferroconvection in a Boussinesq ferrofluid medium in the presence of an imposed magnetic field is described in terms of a simplified model such that

(i) linear results are identical with those for the full problem, and

(ii) finite amplitude solution is exact to second order in the amplitude of the motion and appears to be qualitatively correct for larger amplitudes.

This is achieved with a fifth-order system of ordinary differential equations which possesses both periodic and steady solutions and allows subcritical convection. In the investigation, Brinkman-Forchheimer-extended Darcy is used. Also, the effective viscosity is considered to be different from viscosity of the ferrofluid.

The instability of the system is controlled by the thermal Rayleigh number $R$ and the magnetic Rayleigh number $R_{m}$, both of which provide effective measures of the temperature difference

a

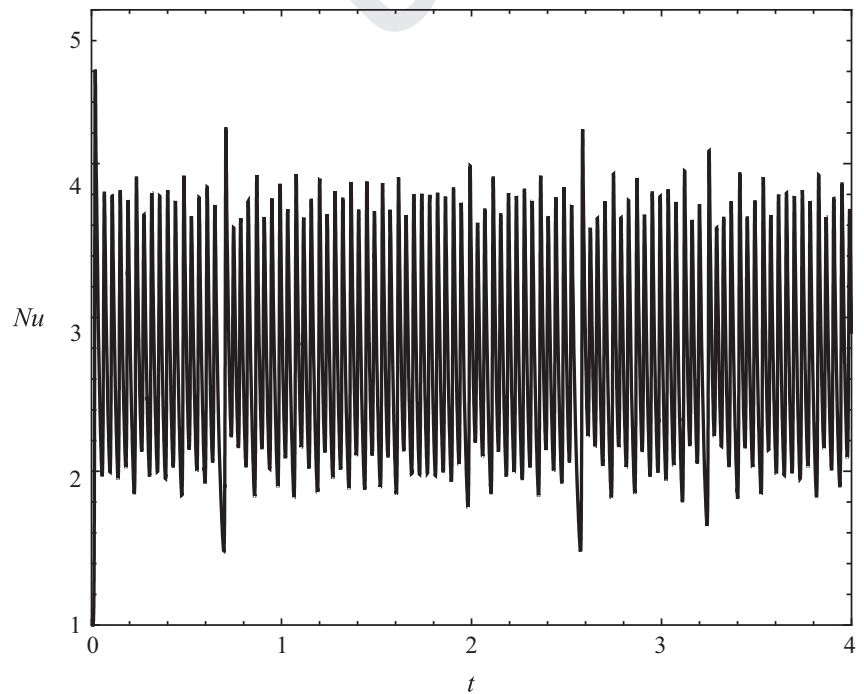

Fig. 8. Variation of Nusselt number with time $t$ for two of values of (a) $D a^{-1}=10$ and (b) $D a^{-1}=25$, when $\operatorname{Pr}=7, T a=100, R_{m}=40 \Lambda=2$ and $R=80000$. across the fluid layer. The thermal Rayleigh number $R$ is proportional to the temperature gradient while the magnetic Rayleigh number $R_{m}$ is proportional to the square of the temperature gradient. Although $R$ can be positive or negative, $R_{m}$ can never be negative. The sign of $R$ indicates which boundary is hotter. When $R>0$, the lower boundary is hotter and both the gravitational buoyancy force and the magnetic body force are destabilizing. When $R<0$, the upper boundary is hotter, the gravitational buoyancy force is stabilizing and only the magnetic body force can produce instability. For a particular value of thermal Rayleigh number $R$ a curve of marginal stability, $R_{m}^{S}$ is found in the present study (Fig. 1).

For given values of thermal Rayleigh number $R$, Taylor number $T a$, viscosity ratio $\Lambda$ and the inverse Darcy number $D a^{-1}$, (29) describes the neutral curves for the onset of ferroconvection. The neutral stability curves in the $\left(R_{m}, a\right)$ - plane for different values of $R$ with $T a=100$ and $T a$ with $R=500$ for a fixed value of $\operatorname{Pr}=10$, $D a^{-1}=100$ and $\Lambda=2$ are shown in Fig. 2(a) and (b), respectively. Fig. 3(a) and (b) respectively display the neutral curves for different values of $\Lambda$ with $D a^{-1}=100$ and $D a^{-1}$ with $\Lambda=2$ for $T a=100$ and $R=500$. The unstable states lay above each neutral stability curve and the figures show the upward concave shape of the neutral stability curves, typical of stability problems of the Rayleigh-Bénard type. From the figures it is evident that the stability region for the system heating from above increases

a

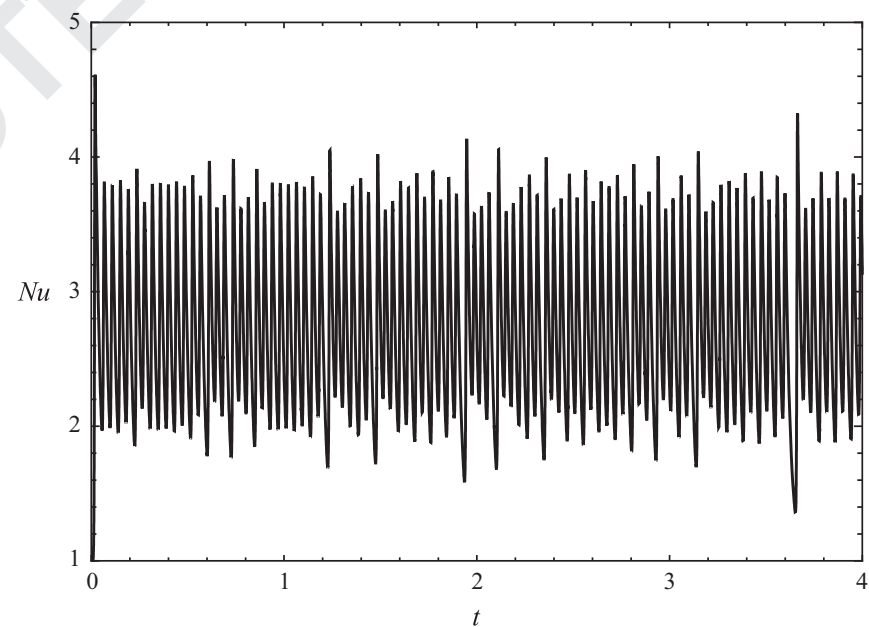

b

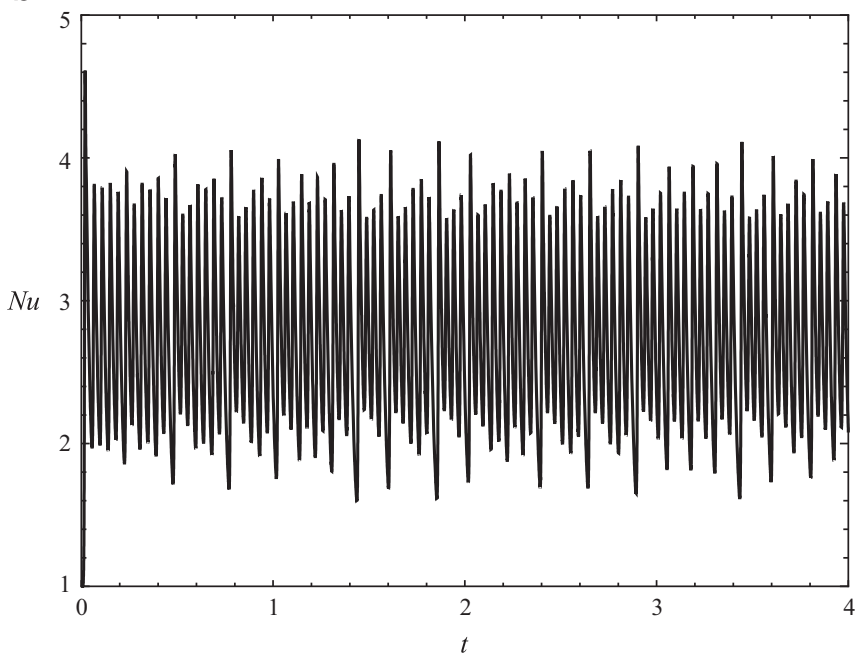

Fig. 9. Variation of Nusselt number with time $t$ for two of values of (a) $R_{m}=0$ and (b) $R_{m}=70$, when $\operatorname{Pr}=7, T a=100, D a^{-1}=25, \Lambda=2$ and $R=80000$. 
compared to heating from below and the neutral stability curve for $R=0$ corresponds to the case of no buoyancy, and in that case the instability is only due to magnetic forces (Fig. 2(a)). Increase in the values of $\mathrm{Ta}$ (Fig. 2(b)), $\Lambda$ (Fig. 3(a)) and $\mathrm{Da}^{-1}$ (Fig. 3(b)) is to increase the region of stability.

The heat transfer is calculated in terms of Nusselt number $\mathrm{Nu}$. Figures illustrate the variation of $\mathrm{Nu}$ as a function of magnetic Rayleigh number $R_{m}$ for different values of physical parameters. From the figures it is observed that the heat transfer increases with increasing $R_{m}$ indicating the ferrofluids transfer heat more effectively. This is due to an increase in the destabilizing magnetic force which hastens the onset of ferroconvection. Moreover, the value of Nusselt number for negative thermal Rayleigh number (heating from above) is lower than those of positive thermal Rayleigh number (heating from below) as the system is more stabilizing when it is heated from above. Increase in the value of $\mathrm{Ta}$ (Fig. 4), $D a^{-1}$ (Fig. 5) and $\Lambda$ (Fig. 6) is to decrease the value of Nusselt number since effect Coriolis force is to delay the onset of ferroconvection. There is no significant effect of pradult number Pr on Heat Transfer and its effect is to hasten the onset of ferroconvection.

The autonomous system of Eqs. (25a)-(25e) is solved numerically using the Runge-Kutta-Gill method with appropriate initial conditions for different values of the governing parameters and the transient behavior of Nusselt number is demonstrated in Fig. 6(a)

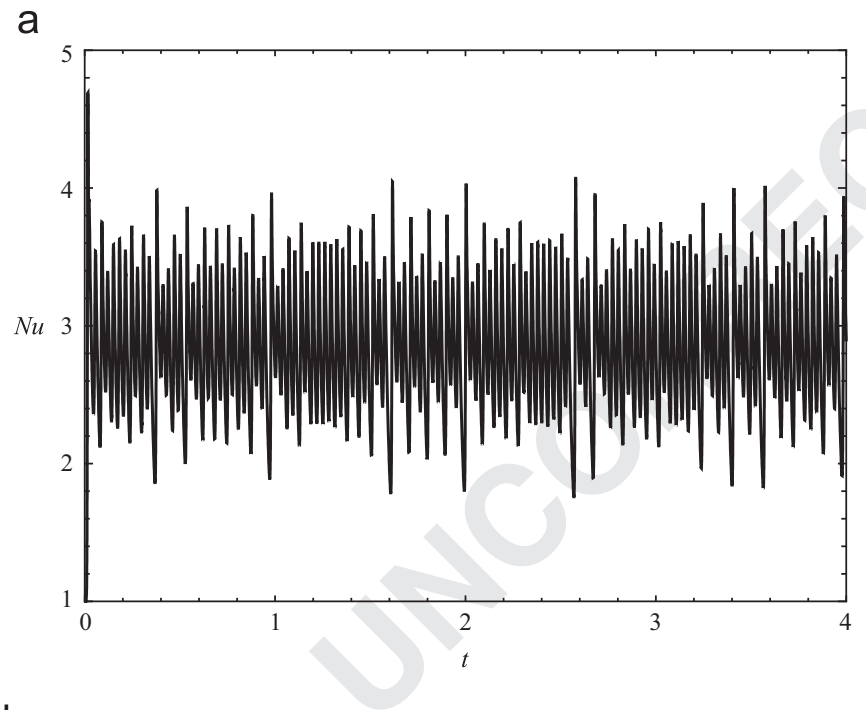

b

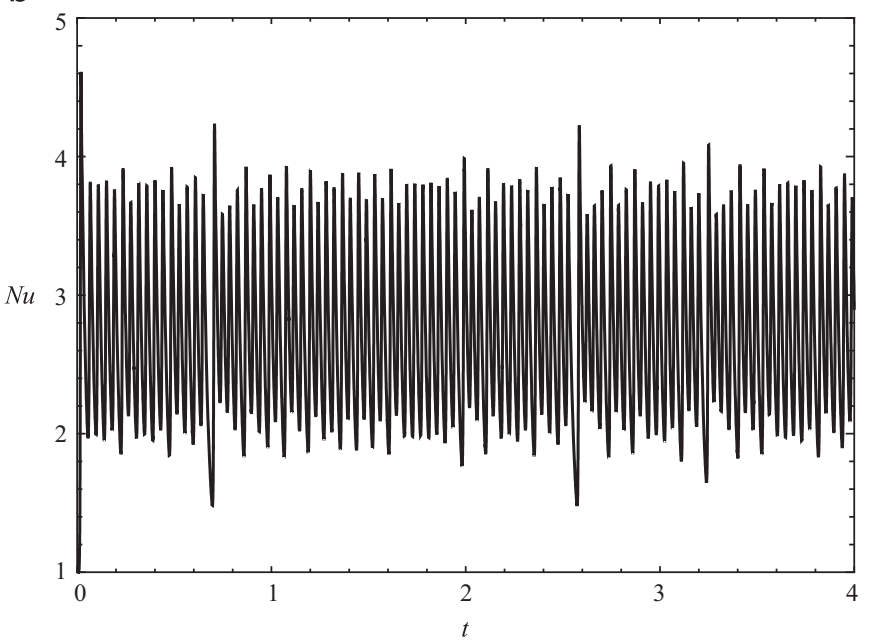

Fig. 10. Variation of Nusselt number with time $t$ for two of values of (a) $\Lambda=1$ and (b) $\Lambda=2$, when $\operatorname{Pr}=7, T a=100, D a^{-1}=25, R_{m}=40 \Lambda=2$ and $R=80000$. and (b), Fig. 7(a) and (b), Fig. 8(a) and (b) and Fig. 9(a) and (b), reveal that heat source enhances heat transport, whereas heat sink diminishes it. Further, it is important to note that there is an excellent agreement between the values of Nu computed analytically for the steady case and numerical results of unsteady case for large time $t$.

The streamlines of steady convection are presented in Fig. 10(a) and (b), Fig. 11(a) and (b), Fig. 12(a) and (b), Fig. 13(a) and (b), for various values of physical parameters. From the figures it is apparent that the convective cells are shrinking with increasing values of $\mathrm{Ta}$ (Fig. 10(a) and (b), $D a^{-1}$ (Fig. 13(a) and (b)) and $\Lambda$ (Fig. 14(a) and (b)). Besides, the cells get contracted little more when the system is heated from above compared to heating from below case $R$ (Fig. 12(a) and (b)).

a

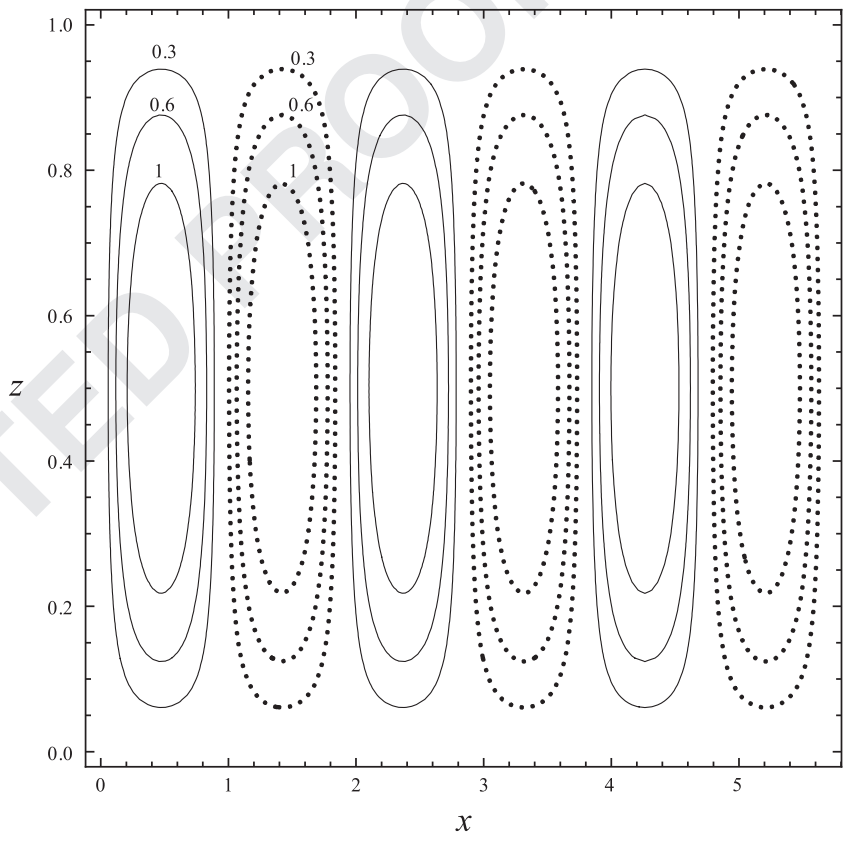

b

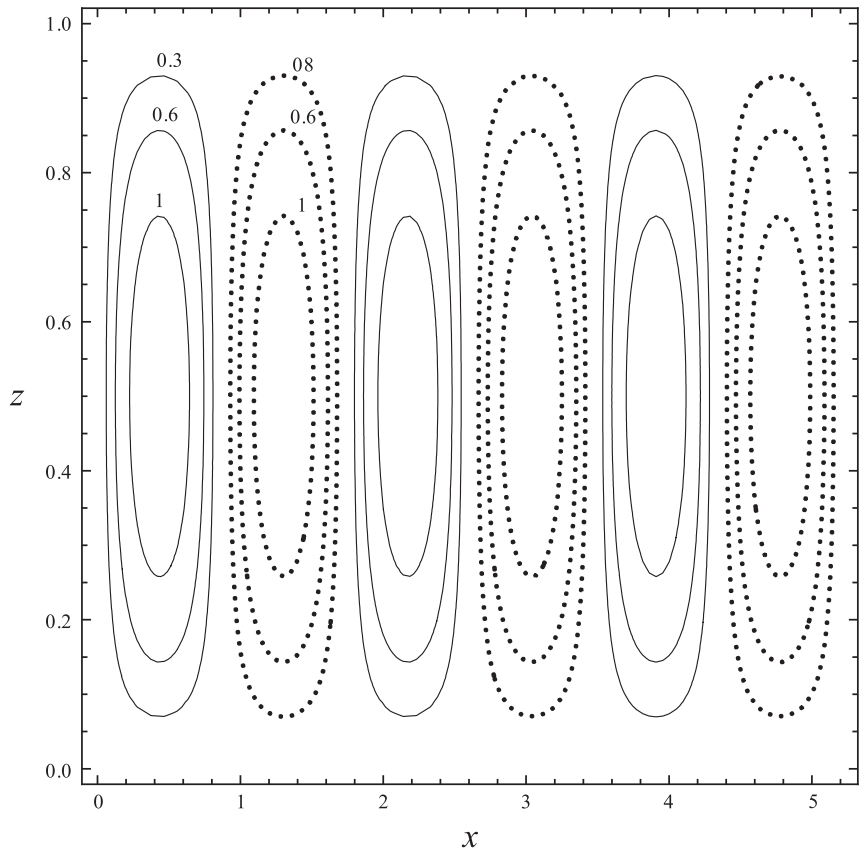

Fig. 11. The streamlines of steady convection for (a) $T a=0$ and (b) $T a=600$ when $\operatorname{Pr}=10, D a^{-1}=25, \Lambda=2, R=500$ and $R_{m}=8000$. 
a

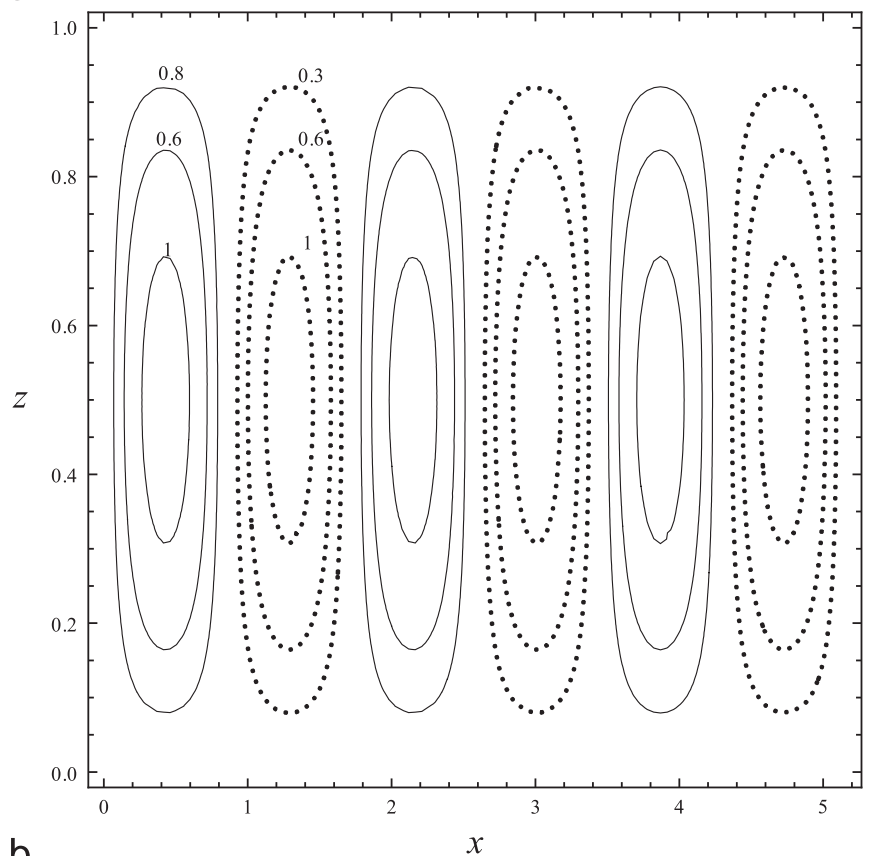

b

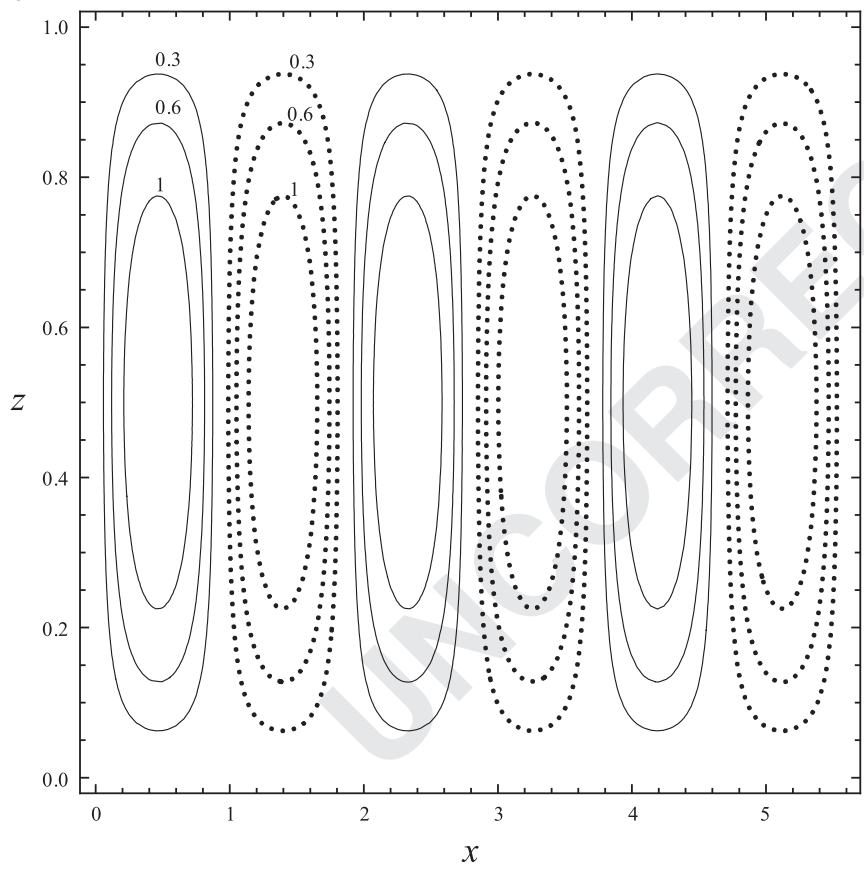

Fig. 12. The streamlines of steady convection for (a) $R=-500$ and (b) $R=500$ when $\operatorname{Pr}=10, D a^{-1}=25, \Lambda=2, T a=100$ and $R_{m}=8000$.

\section{Conclusions}

A weakly nonlinear ferromagnetic convection in a ferrofluid saturated porous medium is studied using Brinkman-extendedDarcy model in a simplified model of two-dimensional convection. This model is exact to second order in the amplitude of the motion and is qualitatively accurate for larger amplitudes. The magnetic Rayleigh number $R_{m}$ is considered as the eigenvalue. From the foregoing study the following conclusions can be drawn.

(i) The model equations considered give linear stability theory results which are identical with those for the full problem. The onset of ferroconvection is delayed when the ferrofluid layer is heated from above compared to heating from below case. a

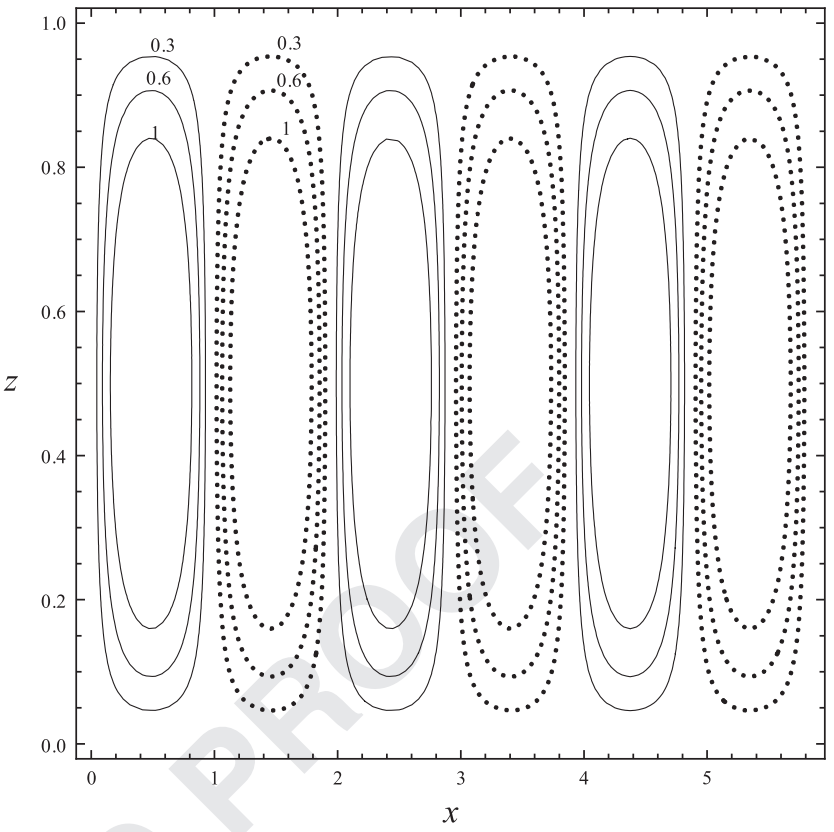

b

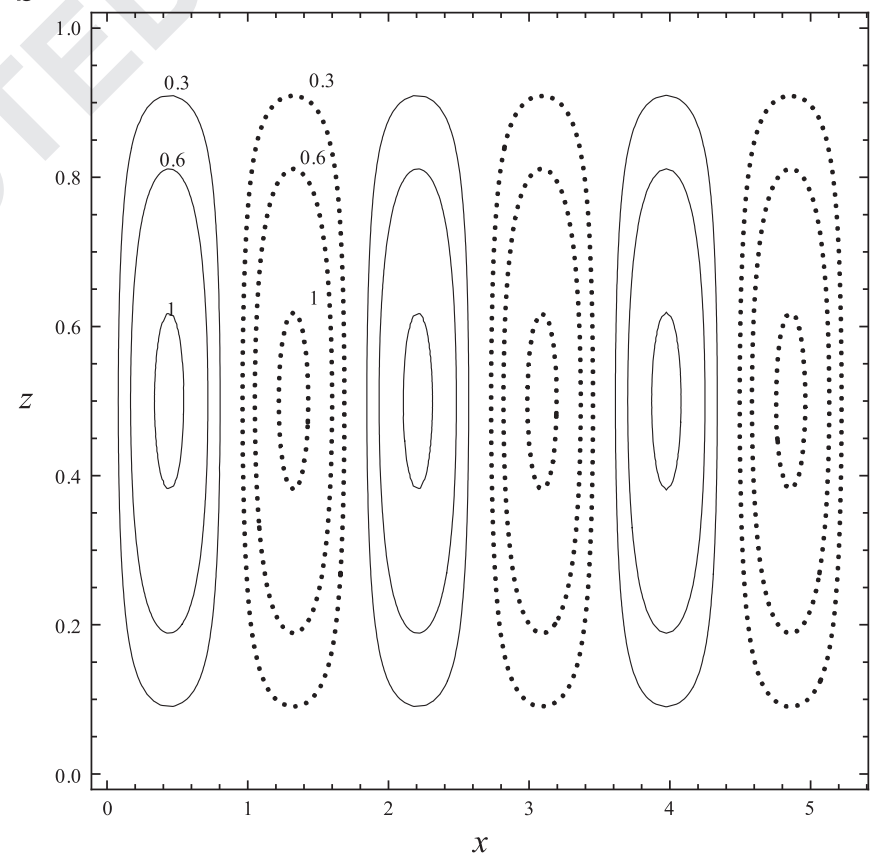

Fig. 13. The streamlines of steady convection for (a) $D a^{-1}=10$ and (b) $D a^{-1}=50$ when $\operatorname{Pr}=10, R=500, \Lambda=2, T a=100$ and $R_{m}=8000$.

(ii) The steady bifurcating equilibrium solution bifurcates only supercritically if the ferrofluid layer is heated from above. But the solution bifurcates subcritically as well when the ferrofluid layer is heated from below. The range of thermal Rayleigh number $R$ beyond which the bifurcation becomes subcritical increases with increasing $\Lambda, D a^{-1}$ and $T a$.

(iii) The global quantity of the heat transfer rate decreases by increasing the Taylor number $T a$.

(iv) The contribution of Nusselt number at the onset of stationary thermomagnetic convection: The Nusselt number increases with increasing $R_{m}$. Increase in the value of $T a, \Lambda$ and $D a^{-1}$ is to decrease. Besides, the numerical result relating to the 


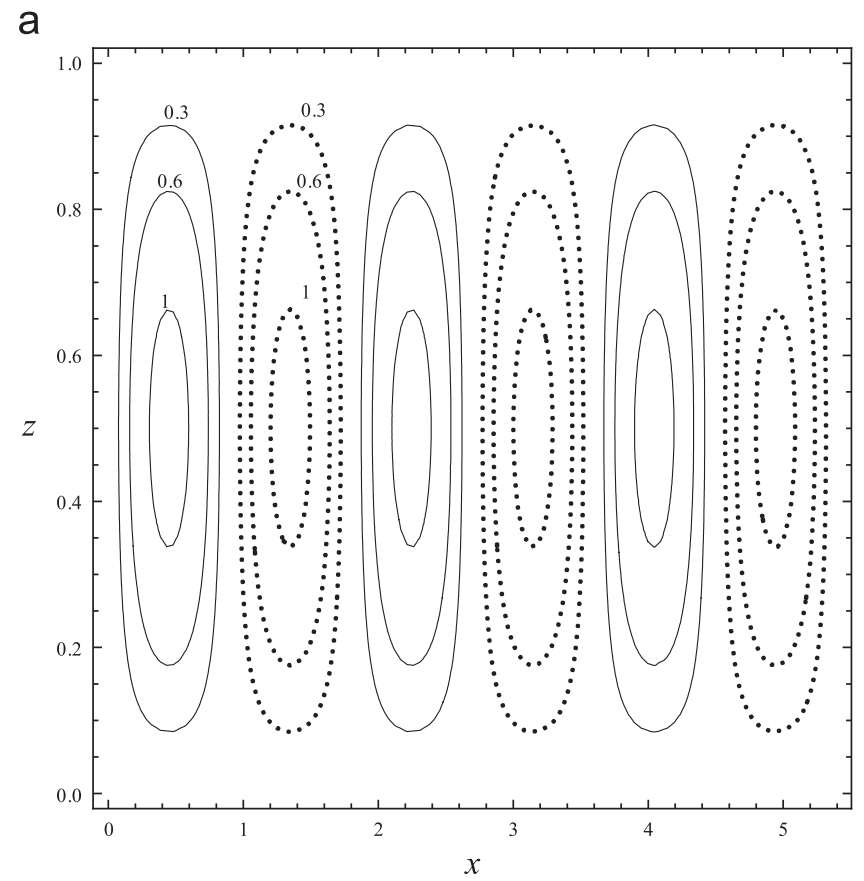

b

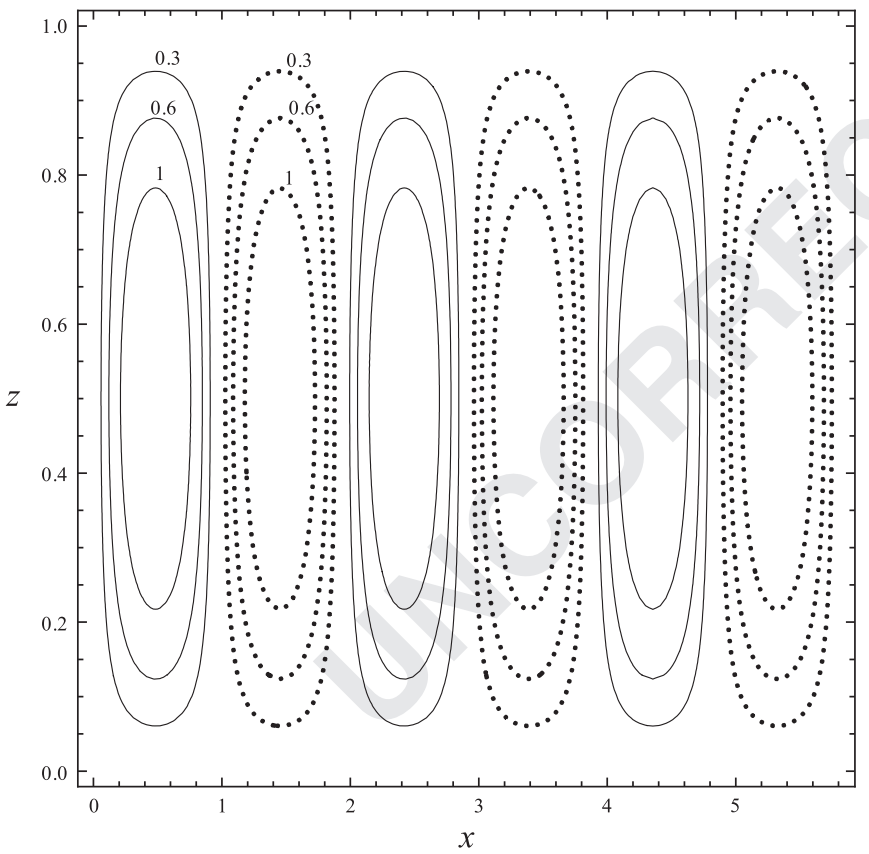

Fig. 14. The streamlines of steady convection for (a) $\Lambda=1$ and (b) $\Lambda=5$ when $\mathrm{Pr}=10, R=500, D a^{-1}=25, T a=100$ and $R_{m}=8000$. transient Nusselt number for large time $t$ compared well with the results of steady state Nusselt number.

(v) The streamlines of steady convection are presented and the convective cells are shrinking with increasing values of $T a, \Lambda$ and $D a^{-1}$, while they get elongated with increasing value of $R$.

\section{References}

[1] S.E. Borglin, J. Mordis, C.M. Oldenburg, Experimental studies of the flow of ferrofluid in porous media, Trans. Por. Med. 41 (2000) 61-80.

[2] C.M. Oldenburg, S.E. Borglin, G.J. Moridis, Numerical simulation of ferrofluids flow for subsurface environmental engineering applications, Trans. Por. Med. 38 (3) (2000) 319-344.

[3] J. Schwab, Thermal convection in ferrofluids under a free surface, J. Magn. Magn. Mater. 85 (1990) 199-202.

[4] P.J. Blenerhasset, F. Lin, P.J. Stiles, Heat transfer through strongly magnetized ferrofluids, Proc. R. Soc. London, Ser. A 433 (1991) 165-177.

[5] C.L. Russell, P.J. Blennerhassett, P.J. Stiles, Supercritical analysis of strongly nonlinear vortices in magnetized ferrofluids, Proc. R. Soc. London, Ser. A 455 (1998) 23-67.

[6] P.N. Kaloni, J.X. Lou, Weakly nonlinear instability of a ferromagnetic fluid rotating about a vertical axis, J. Magn. Magn. Mater. 284 (2004) 54-68.

[7] Sunil, Amit Mahajan, A non-linear stability analysis for magnetized ferrofluid heated from below, Proc. R. Soc. London, Ser. A 464 (2008) 83-98.

[8] D. Laroze, P.G. Siddheshwar, H. Pleiner, Chaotic convection in a ferrofluid, Commun. Nonlinear Sci. Numer. Simul. 18 (2013) 2436-2447.

[9] B. Straughan, Global nonlinear stability in porous convection with a thermal non-equilibrium model, Proc. R. Soc. London, Ser. A 462 (2006) 409-418.

[10] Y. Qin, J. Chadam, A non-linear stability problem for ferromagnetic fluids in porous medium, Appl. Math. Lett. 8 (2) (1995) 25-29.

[11] Sunil, Amit Mahajan, A nonlinear stability analysis for thermoconvective magnetized ferrofluid saturating a porous medium, Trans. Por. Med. 76 (2009) 327-343.

[12] Poonam Sharma Sunil, Amit Mahajan, Nonlinear ferroconvection in a porous layer using a thermal non-equilibrium model, Spec. Top. Rev. Porous MediaInt. J. 1 (2) (2010) 105-121.

[13] E. Blums, G. Kronkalns, A. Mezulis, V. Sints, Non-isothermal mass transfer of ferrocolloids through porous membrane, J. Magn. Magn. Mater. 323 (10) (2011) 1334-1337.

[14] Jyoti Prakash, On exchange of stabilities in ferromagnetic convection in a rotating porous medium, Int. J. Fluid Mech. Res. 40 (5) (2013).

[15] R. Sekar, K. Raju, R. Vasanthakumari, A linear analytical study of Soret-driven ferrothermohaline convection in an anisotropic porous medium, J. Magn. Magn. Mater. 331 (2013) 122-128.

[16] Jyoti Prakash, Sanjay Gupta, On arresting the complex growth rates in ferromagnetic convection with magnetic field dependent viscosity in a rotating ferrofluid layer, J. Magn. Magn. Mater. 345 (2013) 201-207.

[17] B. Straughan, Global nonlinear stability in porous convection with a thermal non-equilibrium model, Proc. R. Soc. London, Ser. A 462 (2006) 409-418.

[18] R. Sekar, G. Vaidyanathan, A. Ramanathan, The ferroconvection in fluids saturating a rotating densely packed porous medium, Int. J. Eng. Sci. 31 (2) (1993) 241-250.

[19] C.E. Nanjundappa, I.S. Shivakumara, H.N. Prakash, Penetrative ferroconvection via internal heating in a saturated porous layer with constant heat flux at the lower boundary, J. Magn. Magn. Mater. 324 (2012) 1670-1678.

[20] C.E. Nanjundappa, H.N. Prakash, I.S. Shivakumara, Jinho Lee, Effect of temperature dependent viscosity on the onset of Bénard-Marangoni ferroconvection, Int. Commun. Heat Mass Trans 51 (2014) 25-30.

[21] G. Veronis, Motions at subcritical values of the Rayleigh number in a rotating fluid, J. Fluid Mech 24 (3) (1966) 545-554 (Jul). 